\title{
Search for New Physics in CP-violating Phenomena at $\mathrm{LHCb}$
}

\section{Johan Blouw ${ }^{* \dagger}$}

Physikalisches Institut of the University of Heidelberg

E-mail: johan.blouwephysi.uni-heidelberg.de

LHCb is an experiment optimised to search for New Physics through measurements of the decays of $\mathrm{B}$ and $\mathrm{D}$ hadrons, in particular those which allow access to CP-violating observables. The principal goals are studies of $\mathrm{B}_{s}$ mixing-induced $\mathrm{CP}$ violation in channels such as $\mathrm{B}_{s} \rightarrow J / \Psi \phi$, a precise measurement of the unitary triangle angle $\gamma$, and the search for $\mathrm{CP}$ violation in the charm sector. Early results will be shown from the 2010 run illustrating the performance of the $\mathrm{LHCb}$ detector. These will be used to predict the expected physics sensitivity in the above topics with the integrated luminosity of $37.7 \mathrm{pb}^{-1}$ that has been recorded by LHCb this year.

The Xth Nicola Cabibbo International Conference on Heavy Quarks and Leptons,

October 11-15, 2010

Frascati (Rome) Italy

\footnotetext{
*Speaker.

On behalf of the LHCb collaboration
} 


\section{Introduction}

The abundance of matter over anti-matter in the universe [1] is a continous puzzle in the Standard Model (SM), since it predicts insufficient CP violation to explain this abundance. Nevertheless, Standard Model predictions of CP-violating observables are consistent with all measurements so far, therefore suggesting a clear need for physics beyond the SM to explain that abundance $[2,3]$.

In the Standard Model, CP violation in flavour changing currents is caused by one single phase in the mixing matrix. This matrix describes the charged current weak interactions of quarks [4]. This so-called Cabbibo-Kobayashi-Maskawa (CKM) matrix is a complex, unitary, $3 \times 3$ matrix which relates the electroweak eigenstates of the quarks with their mass eigenstates. From the unitarity requirement of the CKM matrix 6 orthogonality relations can be derived, which are usually displayed as unitarity triangles in a complex plane.

An example is the unitarity relation

$$
\mathrm{V}_{\mathrm{ub}}^{*} \mathrm{~V}_{\mathrm{us}}+\mathrm{V}_{\mathrm{cb}}^{*} \mathrm{~V}_{\mathrm{cs}}+\mathrm{V}_{\mathrm{tb}}^{*} \mathrm{~V}_{\mathrm{ts}}=0 .
$$

which can be studied using $b \rightarrow c \bar{c} s$ quark-level transitions. The elements $\mathrm{V}_{x y}$ describe the complex coupling strengths of the up-type quarks $(x=u, c, t)$ to the down-type quarks, $(y=d, s, b)$. The angle between the two larger sides $\left(\mathrm{V}_{\mathrm{cb}}^{*} \mathrm{~V}_{\mathrm{cs}}\right)$ and $\left(\mathrm{V}_{\mathrm{tb}}^{*} \mathrm{~V}_{\mathrm{ts}}\right)$ is small:

$$
\beta_{s}=\arg \left[-\frac{\mathrm{V}_{\mathrm{cb}}^{*} \mathrm{~V}_{\mathrm{cs}}}{\mathrm{V}_{\mathrm{tb}}^{*} \mathrm{~V}_{\mathrm{ts}}}\right] \approx \lambda^{2} \eta \approx \arg \left(\mathrm{V}_{\mathrm{ts}}\right)-\pi \approx 0.02
$$

The neutral $\mathrm{B}_{s}$ meson undergoes mixing which can be described by an effective Hamiltonian consisting of two $2 \times 2$ matrices: the mass matrix $M$, and decay matrix $\Gamma$. The mass difference between the mass eigenstates is defined as $\Delta m_{s}=m_{H}-m_{L}$, and the corresponding decay-time difference by $\Delta \Gamma_{s}=\Gamma_{L}-\Gamma_{H}$, where $(L, H)$ indicate the heavy and light mass state. Since the mass eigenstates are not equal to the weak eigenstates, these matrices contain off-diagonal elements, $M_{12}$ and $\Gamma_{12}$. The phase difference between $M_{12}$ and $\Gamma_{12}$ leads to an observable CP-violating phase. In the $\mathrm{SM}$ it is related to $\beta_{s}$ through $\phi_{s} \approx-2 \arg \left(\mathrm{V}_{\mathrm{tb}}^{*} \mathrm{~V}_{\mathrm{ts}}\right)$.

Experimentally, the angle $\phi_{s}$ is measured by evaluating the time-dependent CP asymmetry of the $\mathrm{B}_{s} \rightarrow J / \Psi \phi$ decay which is constructed from the time-dependent decay rates $\Gamma$ for initial $\overline{\mathrm{B}}_{s}$ mesons and $\mathrm{B}_{s}$ mesons to the same final state $f$, where $f$ is a CP eigenstate. It is usually parameterised as

$$
\mathrm{A}_{\mathrm{CP}}(t)=\frac{-\eta_{f} \sin \phi_{s} \sin \left(\Delta m_{s} t\right)}{\cosh \left(\Delta \Gamma_{s} \frac{t}{2}\right)-\eta_{f} \cos \phi_{s} \sinh \left(\Delta \Gamma_{s} \frac{t}{2}\right)},
$$

where $\eta_{f}=+1,-1$ for the $\mathrm{CP}$-even, $\mathrm{CP}$-odd sub sample respectively. The mass-difference between the mass eigenstates of the $\mathrm{B}_{s}^{0}$ meson determines the oscillation frequency of this asymmetry.

Contrary to the $\mathrm{B}_{s}$ case, $\mathrm{CP}$ violation in the $\mathrm{D}^{0}$ system is expected to be negligibly small. This is related to the fact that in this case the mixing proceeds via intermediate states with down-type quarks. In contrast to the case of $\mathrm{B}_{s}$-mixing, here the heaviest participating quark, the b-quark, is strongly suppressed by the CKM-factor $\left|V_{u b} V_{c b}^{*}\right|^{2} /\left|V_{u s} V_{c s}^{*}\right|^{2} \sim \lambda^{8}$, and the GIM mechanism. With this factor, its contribution to D-mixing is only at the percent level, and therefore negligible. In the case of mixing, the mass eigen states $|D 1\rangle,|D 2\rangle$ are composed of linear combinations of the two flavour eigen states $\mathrm{D}^{0}$ and $\overline{\mathrm{D}}^{0},|D 1\rangle=p\left|\mathrm{D}^{0}\right\rangle+q\left|\overline{\mathrm{D}}^{0}\right\rangle$ and $|D 2\rangle=p\left|\mathrm{D}^{0}\right\rangle-q\left|\overline{\mathrm{D}}^{0}\right\rangle$. 


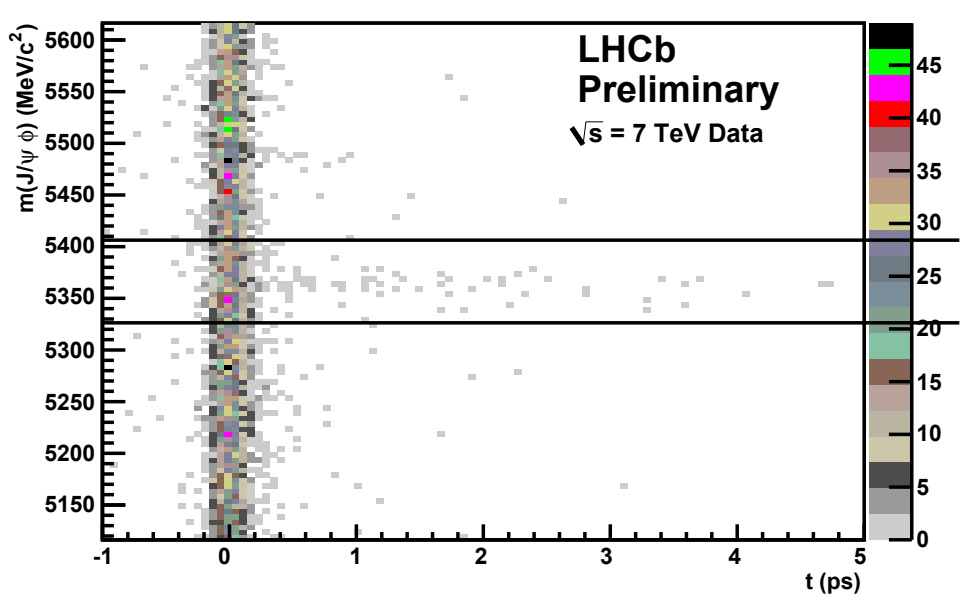

Figure 1: Reconstructed $\mathrm{B}_{s} \rightarrow J / \Psi \phi$ signal vs. proper time in $\mathscr{L} \approx 2 \mathrm{pb}^{-1}$ of data.

This leads to the following relations for the mass difference $(\Delta M)$, decay width $(\Gamma)$ and width difference $(\Delta \Gamma): x=\frac{\Delta M}{\Gamma}$ and $y=\frac{\Delta \Gamma}{2 \Gamma}$. The two decay rates are defined as $A_{f}=\left\langle f|\mathscr{H}| \mathrm{D}^{0}\right\rangle$ and $\bar{A}_{f}=\left\langle f|\mathscr{H}| \overline{\mathrm{D}}^{0}\right\rangle$. Using $\lambda_{f}=\frac{q}{p} \frac{\bar{A}_{f}}{\bar{A}_{f}}$, the decay rate for $\mathrm{D}^{0} \rightarrow f$ can be expressed as

$$
\Gamma\left(\mathrm{D}^{0}(t) \rightarrow f\right)=\frac{1}{4} e^{-\Gamma t}\left|\bar{A}_{f}\right|^{2}\left|\frac{q}{p}\right|^{2}\left[4|\lambda|^{2}+\left(\Delta M^{2}+\frac{\Delta \Gamma^{2}}{4}\right) t^{2}+(2 \Re(\lambda) \Delta \Gamma+4 \mathfrak{I}(\lambda) \Delta M) t\right]
$$

In order to measure possible $\mathrm{CP}$ violation in the D-meson system, the flavour of the meson at the time of its decay has to be determined. For this purpose, the case of the fully reconstructed $\mathrm{D}^{*} \rightarrow \pi_{s} \mathrm{D}^{0}\left(\overline{\mathrm{D}}^{0}\right)$ chain is considered. Here, the charge of the slow pion $\left(\pi_{s}^{ \pm}\right)$is used to tag the flavour of the $\mathrm{D}_{s}$ meson at production time. The doubly-Cabibbo-suppressed decay of the $\overline{\mathrm{D}}^{0}$ into $f=\mathrm{K}^{+} \pi^{-}$is a flavour-eigen state, and hence can be used to determine $\mathrm{CP}$ violation from $\mathrm{D}^{0}-\overline{\mathrm{D}}^{0}$ mixing.

Using Nir's parameterisation [5] of the effects of indirect CP violation, $\lambda=\sqrt{R_{D}} e^{-i(\delta+\phi)}$, and rotating the mass and life-time differences due to the presence of a strong phase $(\delta)$ and a weak phase $(\phi): x^{\prime}=x \cos \delta+y \sin \delta$ and $y^{\prime}=y \cos \delta-x \sin \delta$, the time-dependent decay rate can be expressed as

$$
\Gamma\left(\mathrm{D}^{0}(t) \rightarrow \mathrm{K}^{+} \pi^{-}\right)=|\bar{A}|^{2}\left|\frac{q}{p}\right|^{2} e^{-\Gamma t}\left[R_{D}+\sqrt{R_{D}}\left(y^{\prime} \cos \phi-x^{\prime} \sin \phi\right) \Gamma t+\frac{x^{\prime 2}+y^{\prime 2}}{4} \Gamma^{2} t^{2}\right] .
$$

Here, $R_{D}$ is the ratio of amplitude of the Cabibbo-Favoured (CF) decay over the doubly Cabibbo suppressed (DCS) decay. The three terms in expression 1.5 describe the effects of the DCS amplitudes (constant with time), effects from the interference between mixing and DCS amplitudes (linearly with time), and effects from mixing (quadratic with time). 
Theoretically, CP-violating effects in the Standard Model are expected to be on the order of $\mathscr{O}\left(10^{-3}\right)$, hence any measurement of $\mathrm{CP}$ violation larger than this value is a clear signature of physics beyond the standard model.

\section{CP violation in the beauty sector}

At the LHC, the colliding proton beams produce $b \bar{b}$ quark pairs predominantly in the forward and backward direction, hence the forward-angle design of the LHCb detector. The design and detailed description of the spectrometer is given elsewhere [6]. As of writing, the LHC has delivered $\mathscr{L}=42.15 \mathrm{pb}^{-1}$ of colliding proton beams, out of which $\mathrm{LHCb}$ recorded $\mathscr{L}=37.7 \mathrm{pb}^{-1}$ (equivalent to a $89.3 \%$ efficiency).

The LHCb collaboration will measure the phase $\phi_{s}$ using the $\mathrm{B}_{s} \rightarrow J / \Psi \phi$ channel. Here, the flavour of the $\mathrm{B}_{s}$ at creation time needs to be tagged, and the proper time of the decaying $\mathrm{B}_{s}$ so that asymmetry $\mathscr{A}_{\mathrm{CP}}$ can be constructed. For the determination of the flavour of the $\mathrm{B}_{s}$ at decay, the propertime and its oscillation frequency $\Delta m_{s}$ have to be known. The final state of the $\mathrm{B}_{s}$ decay is composed of CP-even and CP-odd components, and will be disentangled using an analysis of the angular distribution of the final state decay products.

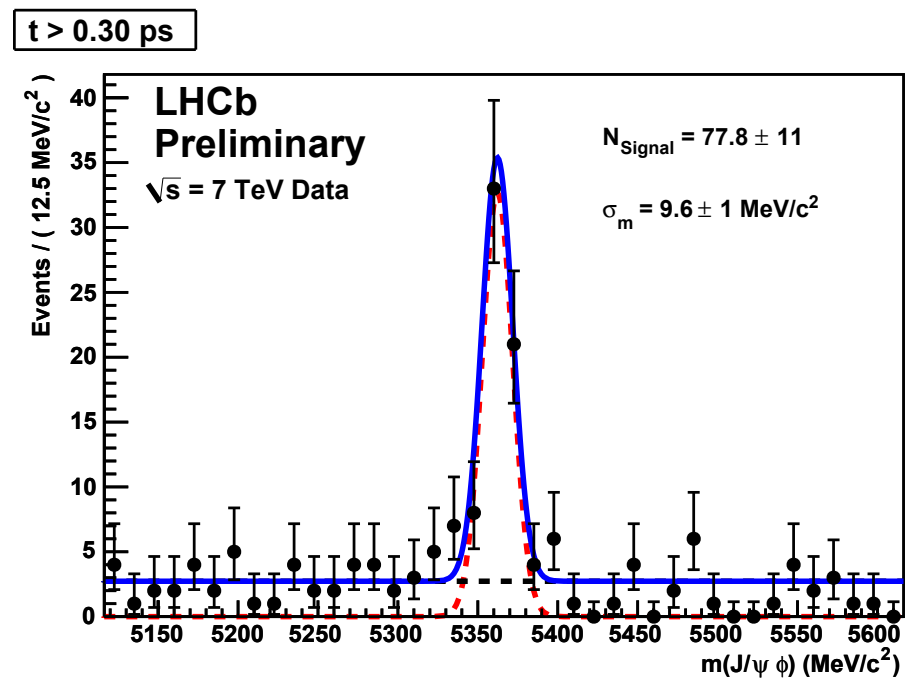

Figure 2: Reconstructed mass for $\mathrm{B}_{s} \rightarrow J / \Psi \phi$ signal for $\tau>0.3 \mathrm{ps}$ in $\mathscr{L} \approx 2 \mathrm{pb}^{-1}$ of data.

In order to study the angular acceptance of the spectrometer, the decay $\mathrm{B}_{d} \rightarrow J / \Psi \mathrm{K}^{*}$ is used; for the proper time resolution, the channels $\mathrm{B}_{d} \rightarrow J / \Psi \mathrm{K}^{*}$ and $\mathrm{B}_{u}^{+} \rightarrow J / \Psi \mathrm{K}^{+}$are useful. The channel $\mathrm{B}_{d} \rightarrow J / \Psi \mathrm{K}_{s}$ is used as a benchmark channel. A coherent set of cuts for the reconstruction of the four $\mathrm{B} \rightarrow J / \Psi X, X \in\left(\phi, \mathrm{K}^{*}, \mathrm{~K}^{+}, \mathrm{K}_{s}\right)$ decays has been developed, based mainly on track- and vertexquality criteria, particle identification criteria and requirements on the transverse momentum of the two muons $P_{t}\left(\mu^{ \pm}\right)>0.5 \mathrm{GeV}$, and the $J / \Psi, P_{t}(J / \Psi)>1.0 \mathrm{GeV}$. In addition, criteria are imposed upon the other particle/resonance (e.g. $P_{t}(\phi)>1.0 \mathrm{GeV}$, and on the reconstructed B meson (impact 


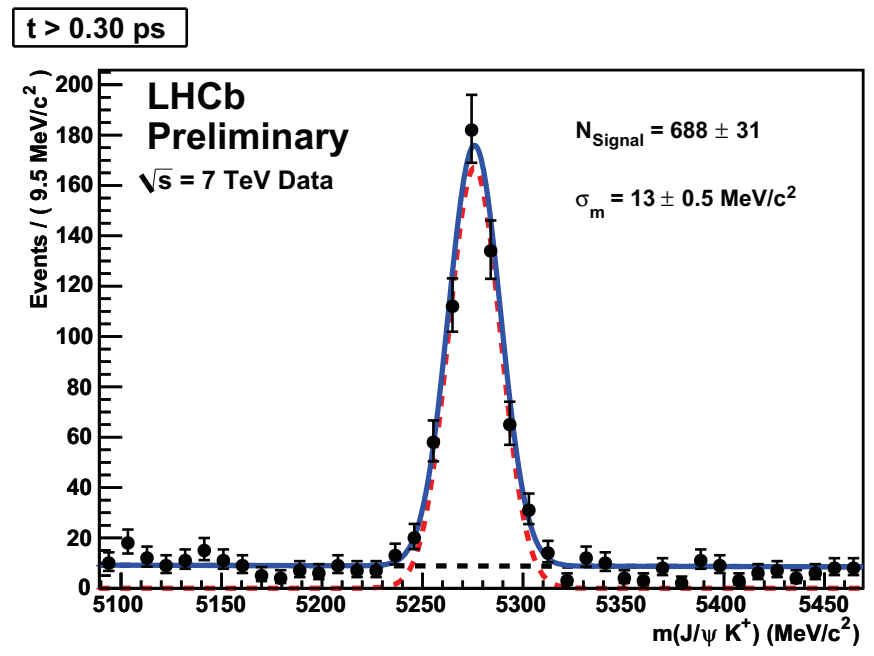

Figure 3: Reconstructed $\mathrm{B}_{u}^{+}$mass from the exclusive decay $\mathrm{B}_{u}^{+} \rightarrow J / \Psi \mathrm{K}^{+}$with the requirement $\tau>0.3 \mathrm{ps}$.

parameter significance and vertex reconstruction). In this way, a mostly lifetime-unbiased selection is achieved.

Using an integrated luminosity of approximately $\mathscr{L} \approx 2 \mathrm{pb}^{-1}$, the $\mathrm{B}_{s} \rightarrow J / \Psi \phi$ channel has been reconstructed. In Figure 1 the reconstructed $\mathrm{B}_{s}$ mass $v s$. proper time is shown. Figure 2 shows the background subtracted signal, for proper time $\tau\left(\mathrm{B}_{s}\right)>0.3$ ps. From this, a yield of $N_{\mathrm{B}_{s}}=38.9 \pm 5.5$ has been determined.

Another example of the result of the analysis of these four channels is the $\mathrm{B}_{u}^{+} \rightarrow J / \Psi \mathrm{K}^{+}$decay. The signal is shown in Figure 3, with the background removed by a requirement on the proper time, $\tau>0.3$ ps. In $\mathscr{L}=3 \mathrm{pb}^{-1}$, a yield $N_{\mathrm{B}_{d}}=688 \pm 31$ events has been found.

The opposite site tagger is being calibrated using in addition to the $\mathrm{B}_{u}^{+} \rightarrow J / \Psi \mathrm{K}^{+}$channel, also the $\mathrm{B}_{d} \rightarrow J / \Psi \mathrm{K}^{* 0}$ channel. As a test, the tagger has been used on $\mathscr{L}=2 \mathrm{pb}^{-1}$ of data with the $\mathrm{B}_{d} \rightarrow \mathrm{D}^{*-} \mu^{+} v$ channel, where the $\mathrm{D}^{*-} \rightarrow \mathrm{D}^{0} \pi^{-}$and the $\mathrm{D}^{0} \rightarrow \mathrm{K}^{+} \pi^{-}$. Here, both the mass difference and the $\mathrm{B}$ proper time were fitted, to result in the first observation of $\mathrm{B}_{d}$ oscillations at LHCb, see Fig. 4. A preliminary value of $\Delta m_{d}=0.530 \pm 0.076 \times 10^{12} \hbar / \mathrm{s}$ was found, where only a statistical error was determined. Note that here, the tuning of the the tagging application had yet to be performed, and also that the proper time calibration is still under way.

\section{CP violation in the charm sector}

$\mathrm{CP}$ violation in the charm sector can be measured in two independent ways. The first method relies on the different time evolution of the components in eq. 1.5. In this case, the decay $\mathrm{D}^{*+} \rightarrow$ $\pi_{s} \mathrm{D}^{0}$ is used, where the slow pion $\left(\pi_{s}\right)$ tags the flavour of the initial $\mathrm{D}^{0}$. For the CP measurement, the wrong-sign (WS) decay $\mathrm{D}^{0} \rightarrow \pi^{-} \mathrm{K}^{+}$is reconstructed. In this decay two amplitudes contribute, namely a diagram where mixing occurred followed by the subsequent Cabibbo-favoured decay to $\mathrm{K}^{-} \pi^{+}$, and the amplitude where no mixing occurred and the $\mathrm{D}^{0}$ decays via the doubly Cabibbosuppressed channel directly into $\mathrm{K}^{-} \pi^{+}$. So far, an integrated luminosity of $\mathscr{L}=124 \mathrm{nb}^{-1}$ has 


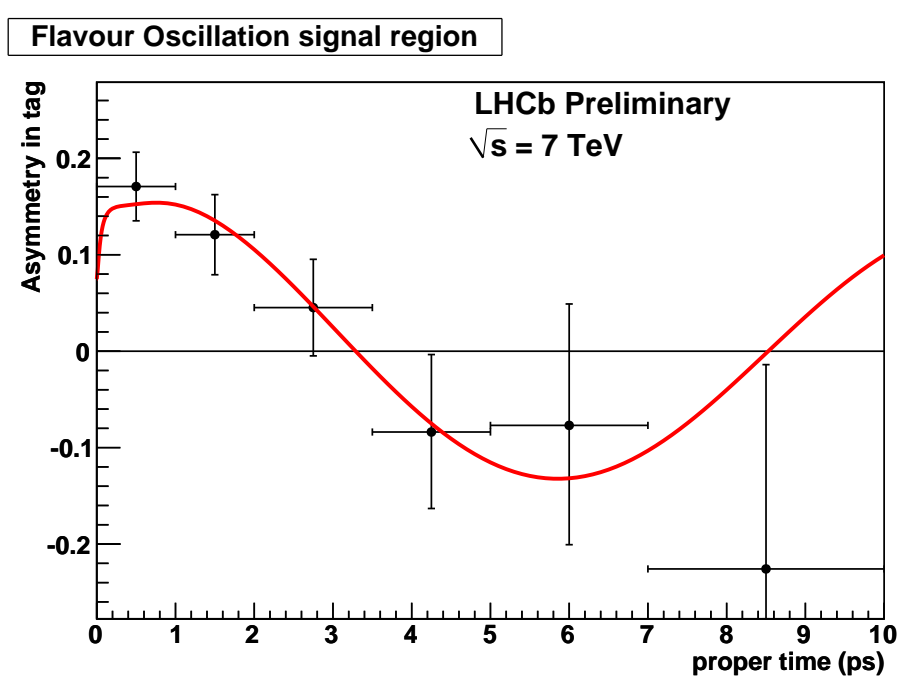

Figure 4: Oscillations of the $\mathrm{B}_{d} \rightarrow \mathrm{D}^{*-} \mu^{+} v_{\mu}$ channel in LHCb.

been analysed. With this small sample, a yield of $N(R S)=17033 \pm 186$ events for the right-sign (RS) decay in the chain $\mathrm{D}^{*+} \rightarrow \pi_{s} \mathrm{D}^{0}\left(\rightarrow \mathrm{K}^{-} \pi^{+}\right)$was found; the observation of the WS decay has yet to be released. In Figure 5(a) the reconstructed right-sign decay is shown. A two-dimensional fit in the $\delta m, m\left(\mathrm{D}^{0}\right)$ plane was performed to derive the yield.

The lifetime difference between CP-even and CP-mixed states can be studied using the decays $\mathrm{D}^{0} \rightarrow \mathrm{K} \pi$ and $\mathrm{D}^{0} \rightarrow \mathrm{KK}$ by means of the quantity known as ' $y_{\mathrm{CP}}$ ':

$$
y_{\mathrm{CP}}=\frac{\tau\left(\mathrm{D}^{0} \rightarrow \mathrm{K}^{-} \pi+\right)}{\tau\left(\mathrm{K}^{-} \mathrm{K}^{+}\right)}-1
$$

In addition, a measurement of the lifetime asymmetry

$$
A_{\Gamma}=\frac{\tau\left(\overline{\mathrm{D}}^{0} \rightarrow \mathrm{K}^{-} \mathrm{K}^{+}\right)-\tau\left(\mathrm{D}^{0} \rightarrow \mathrm{K}^{+} \mathrm{K}^{-}\right)}{\tau\left(\overline{\mathrm{D}}^{0} \rightarrow \mathrm{K}^{-} \mathrm{K}^{+}\right)+\tau\left(\mathrm{D}^{0} \rightarrow \mathrm{K}^{+} \mathrm{K}^{-}\right)}
$$

can be performed using the $\mathrm{D}^{0} \rightarrow \mathrm{KK}$ and $\overline{\mathrm{D}}^{0} \rightarrow \mathrm{KK}$ channels. An example of the reconstructed $\mathrm{D}^{0}$ mass is given in Fig. 5(b).

In a completely independent method, a Dalitz-ratio analysis will be performed using the threebody decay in the channel $\mathrm{D}^{+} \rightarrow \mathrm{K}^{+} \mathrm{K}^{-} \pi^{+}$and its charged conjugate. Here, Dalitz-plot analyses are made for the two decays, after which a Miranda [7] statistic

$$
D_{p_{S_{\mathrm{CP}}}}=\frac{N(i)-\bar{N}(i)}{\sqrt{N(i)+\bar{N}(i)}}
$$

is used to probe for $\mathrm{CP}$ violation. Although this method is very sensitive, and rather robust against e.g. detector-induced asymmetries, it suffers from the fact that it can only establish CP violation, but one can not derive the source. In this analysis, two control channels can be used, namely $\mathrm{D}_{s}^{+} \rightarrow \mathrm{K}^{+} \mathrm{K}^{-} \pi^{+}$and $\mathrm{D}^{+} \rightarrow \mathrm{K}^{+} \pi^{+} \pi^{-}$channels. For an integrated luminosity of $\mathscr{L}=580 \mathrm{nb}^{-1}$ approximately $5000 \mathrm{D}$ events were found. 


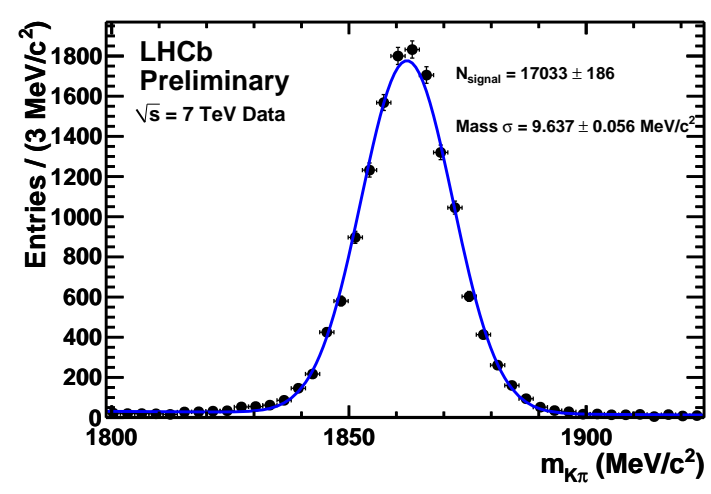

(a)

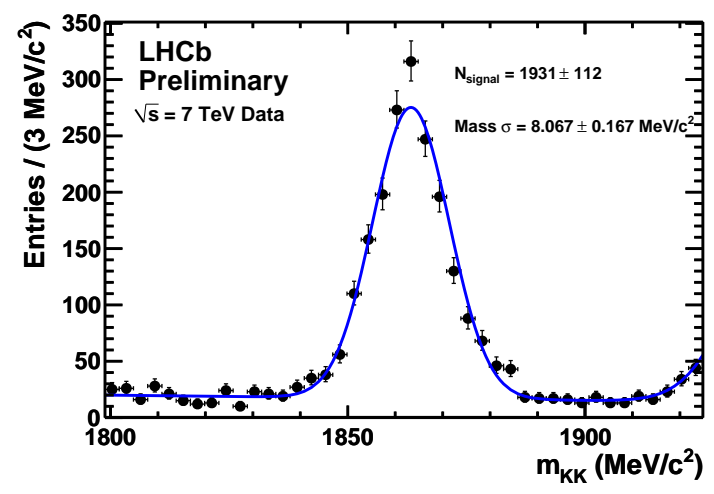

(b)

Figure 5: a) Reconstructed invariant mass of the right-sign decay $\mathrm{D}^{*+} \rightarrow \mathrm{D}^{0}\left(\rightarrow \mathrm{K}^{+} \pi^{-}\right) \pi_{s}$, a mass width of $\sigma=9.6 \mathrm{MeV}$ is found. b) Reconstructed $\mathrm{D}^{0}$ mass using the chain $\mathrm{D}^{*+} \rightarrow \mathrm{D}^{0}\left(\mathrm{D}^{0} \rightarrow \mathrm{KK}\right)$. The fit resulted in a mass width of $\sigma=8.067 \mathrm{MeV}$.

\section{Conclusions}

Thanks to the successful commencement and operation of the Large Hadron Collider, a total of $\mathscr{L}=37.7 \mathrm{pb}^{-1}$ was recorded this year by the LHCb experiment, with an efficiency close to $90 \%$. In many new physics models, new, heavy particles may contribute to processes which proceed through Feynman diagrams of the box- and penguin type. In this way, also possible new CP-violating phases may become visible. In particular, since the Standard Model predicts only small CP violation in the $\mathrm{B}_{s}$ meson sector and negligible $\mathrm{CP}$ violation in the D-meson sector, here measurements of these quantities may be sensitive to new physics. With these data, a yield of $N\left(\mathrm{~B}_{s}\right) \approx 1 \times 10^{3}$ in the $\mathrm{B}_{s} \rightarrow J / \Psi \phi$ channel is expected with a proper time resolution of $\sigma(t) \approx 50-60 \mathrm{fs}$, yielding an error on $\phi_{s}$ of $\sigma\left(\phi_{s}\right) \approx 0.30-0.80 \mathrm{rad}$. With this dataset, LHCb are expecting $\mathscr{O}\left(10^{6}\right)$ events for the $\mathrm{CP}$ violation searches in the charm sector.

\section{References}

[1] H. Murayama, Nucl. Phys. Proc. Suppl. 111 (2002) 136 [arXiv:hep-ph/0208005].

[2] A. D. Dolgov and Y. B. Zeldovich, Rev. Mod. Phys. 53, 1 (1981).

[3] A. D. Dolgov, "Baryogenesis, 30 years after,” arXiv:hep-ph/9707419.

[4] Y. Nir, “CP violation in meson decays,” arXiv:hep-ph/0510413.

[5] Y. Nir, JHEP 0705 (2007) 102 [arXiv:hep-ph/0703235].

[6] A. A. Alves et al. [LHCb Collaboration], JINST 3 (2008) S08005.

[7] I. Bediaga, I. I. Bigi, A. Gomes, G. Guerrer, J. Miranda and A. C. d. Reis, Phys. Rev. D 80 (2009) 096006 [arXiv:0905.4233 [hep-ph]]. 